\title{
Lehr-Lernkontexte in einer transformativen Fakultät
}

\author{
Konzeptionelle Perspektiven
}

\section{Einleitung}

Im Rahmen der Veränderungen der Fakultät Information und Kommunikation der Hochschule der Medien Stuttgart (HdM) (Mildenberger/Vonhof 2020) in Richtung einer transformativen Fakultät, in der die disziplinären Grenzen durchlässiger gestaltet werden sollten, wurden auch Inhalte und Lehr-Lernarrangements in den Curricula der Fakultät überarbeitet. Im Fokus stand dabei die Frage, wie ein zukunftsfähiges Hochschulstudium aussehen muss.

Betrachtet man die Diskussion um die (grundsätzliche) Ausgestaltung von Hochschulcurricula in den letzten Jahren, zeigen sich drei Diskussionsstränge:

- Studierfähigkeit: Welche Kompetenzen brauchen Studierende, um das Studium erfolgreich abschließen und komplexe Lernarrangements im Studienverlauf meistern zu können (van den Berk et al. 2016).

- Curriculum 4.0/21st Century Skills: Welche Kompetenzen gewinnen in einer durch Digitalisierung und andere Zukunftstrends geprägten Wirtschaft und Gesellschaft an Bedeutung und wie sind sie im Hochschulstudium zu adressieren (etwa Autorengruppe Curriculum 4.0 2018).

- Didaktische Umsetzung: Welche didaktischen Szenarien eignen sich für die Unterstützung der Kompetenzentwicklung der Studierenden in den genannten Bereichen.

Alle drei Aspekte wurden bei den Reformbemühungen der Fakultät aufgegriffen und in ein neues Studienmodell sowie ein neues, für alle Studiengänge identisches Strukturmodell des Bachelorstudiums überführt (Tabelle 1).

Tab. 1: Strukturmodell des neuen Studienkonzepts der Fakultät luK (eigene Darstellung).

\begin{tabular}{lll}
\hline $\begin{array}{l}\text { Traditionelles Fachstudium } \\
\text { mit deutlicher Stärkung wis- }\end{array}$ & $\begin{array}{l}\text { Methodenstudium: Wissen- } \\
\text { schaftliche Grundlagen }\end{array}$ & $\begin{array}{l}\text { Schlüsselkompetenzen als in- } \\
\text { tegraler Bestandteil des Studi- }\end{array}$ \\
$\begin{array}{l}\text { senschaftlich methodischer } \\
\text { Elemente (10 ECTS - Sem. } 1 \text { und } 2)\end{array}$ & ums (15 ECTS - Sem. 2-4) \\
$\begin{array}{l}\text { Projektphase: Integratives Studium von Fach- und Schlüsselkompetenzen durch projektori- } \\
\text { entierte Lehr-Lernformen (35 ECTS - Sem. } 6 \text { und 7) }\end{array}$ \\
\hline
\end{tabular}

๑ Open Access. (C) 2020 Michael Burmester und Tobias Seidl, published by De Gruyter. (⿶) Br-Nc-ND This work is licensed under the Creative Commons Attribution-NonCommercial-NoDerivatives 4.0 License. 
Im Folgenden werden die mit der Studienreform im Wintersemester 2016/17 neu eingeführten übergreifenden Bereiche detaillierter dargestellt.

\section{Methodenstudium: Modul Wissenschaftliche Grundlagen}

Das übergreifende Ziel ist es, Studierende in die Lage zu versetzen, selbständig zukünftige Herausforderungen von Gesellschaft und Wirtschaft bewältigen zu können. Innovative Lösungen lassen sich vor allem vor dem Hintergrund forschender und wissenschaftlich fundierter Vorgehensweisen umsetzen. Vor diesem Hintergrund wurde das Modul Wissenschaftliche Grundlagen konzipiert. Um genügend Zeit und Kapazität zu haben, wurde das Modul über die Laufzeit von zwei Semestern mit einem Gesamtumfang von 10 ECTS geplant. Mit forschendem Lernen als Grundprinzip verfolgt dieses Modul folgende Ziele:

- Wissenschaft und Forschung soll als praktisches Mittel des Lösens von relevanten Problemen verstanden werden.

- Forschung dient dem kontinuierlichen Wissensaufbau und ermöglicht, zukünftigen Herausforderungen methodisch begegnen zu können.

- Wissenschaft und Forschung sollen praktisch angewendet und erlebt werden.

- Für das folgende disziplinäre und interdisziplinäre Studium soll Faszination für Wissenschaft geweckt und eine Forschungskultur an der Fakultät etabliert werden.

An dem zweisemestrigen Modul nehmen Erst- und Zweitsemester aller vier Bachelor-Studiengänge der Fakultät (Informationsdesign, Informationswissenschaften, Online-Medien-Management sowie Wirtschaftsinformatik und digitale Medien) teil. Im Wintersemester beginnen etwa 180 und im Sommersemester etwa 150 Studierende mit dem Studium. Somit studieren in diesem Modul immer etwa 330 Studierende gleichzeitig.

Damit von Beginn an die Studierenden interdisziplinäre Zusammenarbeit einüben können, werden fünfköpfige studentische Arbeitsgruppen aus den vier Studiengängen gebildet. Die so entstehenden 66 studentischen Arbeitsgruppen werden in 11 Seminargruppen zusammengefasst, die jeweils von Professorinnen und Professoren betreut werden. Begleitend vermittelt eine Vorlesung im ersten und zweiten Semester die jeweils relevanten theoretischen Inhalte (Tabelle 2). 
Tab. 2: Vorlesungsinhalte im Modul Wissenschaftliche Grundlagen.

\begin{tabular}{ll}
\hline Vorlesungsinhalte im ersten Semester & Vorlesungsinhalte im zweiten Semester \\
\hline - Wissenschafts- und Erkenntnistheoretische & - Generieren und Formulieren von Hypothesen \\
Grundlagen & - Grundlagen und Methoden quantitativer Da- \\
- Recherche & tenerhebung \\
- Grundlagen und Methoden explorativer und & - Erstellung eines Forschungsdesigns und Stu- \\
qualitativer Forschung & diendurchführung \\
- Erstellung eines Forschungsdesigns und & - Grundlagen deskriptiver und hypothesenprü- \\
Studiendurchführung & fender Statistik \\
- Inhaltsanalytische Auswertung & - Dokumentation wissenschaftlicher Ergebnis- \\
- Wissenschaftliche Dokumentation und & se und Verfassen eines wissenschaftlichen \\
Erstellung eines Posters & Artikels \\
\hline
\end{tabular}

Im Rahmen eines Dies Academicus findet zu Beginn jedes Semesters eine Fakultätskonferenz statt, die den Studierenden Kommunikation im Rahmen der Wissenschaft näherbringen soll. Eingeladen sind alle Studierenden und Dozierenden der Fakultät. Hauptakteurinnen und -akteure sind die Studierenden des Moduls Wissenschaftliche Grundlagen. Die Konferenz beginnt mit einem Vortrag aus den Forschungsgruppen der Fakultät, um einen Einblick in die professionelle Forschung der Fakultät zu geben. Die Zweitsemester präsentieren dann ihre Poster als Kurzpräsentation von einer Minute pro Poster im Rahmen einer sogenannten Poster-Madness. Im Anschluss daran können die Poster betrachtet und mit den jeweiligen studentischen Arbeitsgruppen diskutiert werden.

Die Erstsemester können so bereits erfahren, was sie in der Veranstaltung erwartet und die Zweitsemester starten mit Erkenntnissen aus den Diskussionen in die zweite Hälfte des Moduls. Zudem wird damit für die Zweitsemester ein typisches Kommunikationsformat der Wissenschaft eingeübt und erlebbar gemacht. Zur Konferenz gehört auch, dass ein Best Paper Award vergeben wird. Aus etwa 150 bis 180 wissenschaftlichen Artikeln, die als Prüfungsleistung abgegeben wurden, wird ein erster und zweiter Preis verliehen.

Das Fazit aus sechs Semestern Erfahrung fällt ambivalent aus. Auf der Positivseite kann verbucht werden,

- dass die praktische Anwendung von Forschungsmethoden im Rahmen von für Studierende relevanten Fragestellungen Vorteile und große Potenziale hat,

- dass es sich als sinnvoll erwiesen hat, die zu vermittelnden Kompetenzen im Rahmen eines zweisemestrigen Moduls mit 10 ECTS im ersten und zweiten Semester zu platzieren, 
- dass die vermittelten Kompetenzen von Studierenden im weiteren Verlauf des Studiums benötigt werden und selbständig angewendet werden können.

Neben diesen positiven Aspekten zeigten sich jedoch auch einige Probleme und Verbesserungspotenziale:

- Interdisziplinäre studentische Arbeitsgruppen: Die Studierenden haben im ersten Semester noch keine ausreichende Vorstellung von den Stärken und Schwächen der eigenen Disziplin. Erst, wenn der eigene Studiengang etwas besser kennengelernt wurde, machen interdisziplinäre studentische Arbeitsgruppen Sinn (etwa zum Ende des zweiten Semesters).

- Erarbeiten wissenschaftlicher Fragestellungen: Selbst mit Unterstützung durch Dozierende fällt das Finden und das Formulieren wissenschaftlicher Fragestellungen gerade im ersten Semester schwer. Die Freiheitsgrade waren zu hoch und eine schrittweise Hinführung zu einer Fragestellung ist erforderlich.

- Notwendigkeit statistischer Hypothesenprüfung wird nicht erkannt: Gerade Inferenzstatistik fiel den Studierenden schwer, da die Notwendigkeit im Rahmen des eigenen Studiums oft nicht gesehen wurde. Wichtiger erscheint es, einen eher spielerischen Zugang zum Umgang mit quantitativen Daten mit Mitteln der deskriptiven Statistik einzuführen.

- Veranstaltungskonzeption als vollständiger wissenschaftlicher Prozess: Die Annahme, dass den Studierenden ein wissenschaftlicher Prozess anhand von einer explorativen qualitativen und einer hypothesenprüfenden quantitativen Studie zu vermitteln wäre, ist zu idealistisch. Besser ist es, den wissenschaftlichen Prozess in kleinere Schritte und Aufgaben zu zerlegen und mit den Studierenden zu erarbeiten. Erst im zweiten Semester können dann die notwendigen Wissenselemente zu einer empirischen Studie zusammengeführt werden.

- Wissenschaft und Forschung als Konzepte von „gestern“: Die Bezeichnung eines Studieninhalts als „Wissenschaft“ geht offenbar an der Lebenswirklichkeit von Studierenden vorbei. Bezeichnungen wie beispielsweise „Data Analytics“ wirken sehr viel zeitgemäßer. Somit scheint es notwendig, sich auch über die Bezeichnung von Inhalten Gedanken zu machen.

Vor dem Hintergrund dieser Erkenntnisse wurde das Konzept des Moduls überarbeitet und mit Beginn des Wintersemesters 2019/2020 neu gestartet. Diese Erfahrungen zeigen auch, wie wichtig ein iteratives und entwicklungsorientiertes Vorgehen im Bereich der Curriculumsentwicklung ist (Seidl/Vonhof 2017). 


\section{Schlüsselkompetenzmodule}

Das dezidierte Schlüsselkompetenzstudium findet in drei Pflichtmodulen mit jeweils 5 ECTS in den Semesterstufen 2-4 statt. Bei der Ableitung der Lernziele wurde sowohl die Studierfähigkeit als auch die Vorbereitung der Studierenden auf die Herausforderungen der dynamischen technologischen, wirtschaftlichen und gesellschaftlichen Entwicklung in den Blick genommen (Seidl 2017). Die drei Module folgen alle dem gleichen schematischen Aufbau:

- verpflichtende Kopfveranstaltung des Moduls (2 ECTS),

- Wahlveranstaltung innerhalb des Kompetenzbereichs des Moduls (2 ECTS),

- E-Portfolio und Schlüsselkompetenzkolloquium (1 ECTS).

Die inhaltliche Ausgestaltung der Module verdeutlicht Tabelle 3. Im Mittelpunkt der Schlüsselkompetenzmodule steht die Entwicklung beziehungsweise Verbesserung metakognitiver Strategien. Daneben soll Handlungskompetenz im überfachlichen Bereich erworben werden. Die Lehrenden verknüpfen dafür die Inhalte der Veranstaltungen immer mit aktuellen Herausforderungen in zeitgleich stattfindenden fachspezifischen Veranstaltungen und der Lebensrealität der Studierenden. Damit wird den Studierenden eine wichtige Folie für Reflexionsprozesse und das Ausprobieren neuer Handlungsstrategien geboten. Dieses Setting ermöglicht situiertes und nachhaltiges Lernen. Die Einbindung eines Wahlbereiches in die Pflichtmodule erlaubt es den Studierenden zudem, ihre eigenen Schwerpunkte zu setzen und ihren eigenen Lernweg im Schlüsselkompetenzbereich aktiv zu gestalten.

Tab. 3: Aufbau der einzelnen Module des Bereichs Schlüsselkompetenzen.

\begin{tabular}{|c|c|c|c|}
\hline Modultitel & Tools for Working & Ways of Working & $\begin{array}{l}\text { Working in a Media } \\
\text { World }\end{array}$ \\
\hline Kopfveranstaltung & Projektmanagement & $\begin{array}{l}\text { Intercultural Skills/ } \\
\text { Communication }\end{array}$ & Medienrecht \\
\hline \multirow[t]{3}{*}{ Wahlveranstaltungen } & $\begin{array}{l}\text { Projektmanagement } \\
\text { Vertiefung }\end{array}$ & Kommunikation & $\begin{array}{l}\text { Ethik und Verantwor- } \\
\text { tung }\end{array}$ \\
\hline & Kreativität & Führung \& Teamarbeit & $\begin{array}{l}\text { Datenschutz und Da- } \\
\text { tensicherheit: Kunden- } \\
\text { datenverarbeitung im } \\
\text { Unternehmen }\end{array}$ \\
\hline & Selbstmanagement & Moderation & $\begin{array}{l}\text { Datenschutz und Da- } \\
\text { tensicherheit: Daten- } \\
\text { schutz und Internet }\end{array}$ \\
\hline Kolloquium & $\begin{array}{l}\text { Kolloquium Tools for } \\
\text { Working }\end{array}$ & $\begin{array}{l}\text { Kolloquium Ways of } \\
\text { Working }\end{array}$ & $\begin{array}{l}\text { Kolloquium Working in } \\
\text { a Media World }\end{array}$ \\
\hline
\end{tabular}


Kernelement der Module ist ein alle Veranstaltungen umfassendes E-Portfolio, das zur studienbegleitenden Reflexion des Lernprozesses dient und dessen Bearbeitung wesentlicher Teil der Lern- und Prüfungsanforderung ist. Im semesterabschließenden Kolloquium wird das gesamte Semester auf der Grundlage des Portfolios reflektiert und die weitere Kompetenzentwicklung geplant. Ziel des Einsatzes von E-Portfolio und Kolloquium sind im Detail:

- die Förderung einer bewussteren Gestaltung des Studiums,

- die gezielte Steuerung des Kompetenzerwerbs durch die Studierenden,

- die Steigerung der Reflexionsfähigkeit sowie

- der Erwerb wichtiger Medienproduktionskompetenz.

Im Wintersemester 2017/18 wurde das Konzept umfassend evaluiert $(\mathrm{N}=226$, Rücklaufquote $=90,4 \%$; Schütz-Pitan et al. 2019). Die Ergebnisse weisen darauf hin, dass das Ziel der Steigerung der Reflexionsfähigkeit erreicht wird.

Portfolio und Kolloquium sind durch klare Arbeitsaufträge strukturiert. In den beiden Lehrveranstaltungen jedes Moduls erhalten die Studierenden Reflexionsaufgaben, die sie parallel zur Veranstaltung im E-Portfolio bearbeiten (Buhl/Seidl/Zeiner 2019). Am Ende des Semesters stellen alle Studierende in einer Kleingruppe (drei Studierende plus ein/e Lehrende/r) ihre Semesterreflexion vor (= Kolloquium). Im Kolloquiumsgespräch nimmt die Lehrperson eine coachende Haltung ein und gibt den Studierenden konstruktives Feedback zu folgenden Aspekten:

1. der von ihm/ihr berichteten Kompetenzentwicklung,

2. den von ihm/ihr geplanten weiteren Schritten im Lernprozess,

3. zur Qualität der präsentierten Reflexion.

Der Aspekt 1 soll primär die Selbstwirksamkeit der Studierenden fördern. Bei Aspekt 2 werden den Studierenden unter Umständen praktische Hinweise zur Gestaltung des weiteren Lernprozesses mit auf den Weg gegeben. Aspekt 3 soll die Studierenden unterstützen, die Tiefe der Reflexion bei der Arbeit mit dem E-Portfolio kontinuierlich zu steigern (insgesamt arbeiten die Studierenden mindestens drei Semester mit dem Instrument). Die technische Umsetzung des E-Portfolios wird in Form von individuellen Wordpress-Seiten realisiert.

Eine Bewertung und Rückmeldung zur Reflexion und den Arbeitsergebnissen der Studierenden erfolgt ausschließlich unter formativen Aspekten. Auf eine summative Bewertung wird bewusst verzichtet. Für das Erhalten des Leistungsnachweises ist alleinige Voraussetzung, dass die Reflexion in hinreichendem Maße ausgearbeitet wurde (Schaper/Hilkenmeier 2013). Daneben muss sowohl in der Pflicht- wie auch in der Wahlveranstaltung des Moduls eine komplexe Lernaufgabe erfolgreich absolviert werden, die in realitätsnahe Hand- 
lungszusammenhänge eingebettet ist. Auch hierzu erhalten die Studierenden eine formative Rückmeldung. Das Modul ist (unbenotet) bestanden, wenn alle drei Prüfungsteile (Pflicht-, Wahlveranstaltung, Portfolio/Kolloquium) in hinreichendem Maße ausgearbeitet wurden.

Die Erfahrungen zeigen, dass der Verzicht auf summatives Prüfen in den Modulen eine lernförderliche Fehlerkultur begünstigt und die Motivation, sich auf neue und unsichere Handlungsweisen einzulassen, erhöht. So weisen etwa auch Wildt und Wildt darauf hin, dass eine

durchgehende Orientierung an (End-)Noten das Lernen im Sinne eines selbstkontrollierten Lernens und die intrinsische Motivation zur Bearbeitung des Gegenstandes eher beschädigt als fördert (Wild/Wild 2011, 30).

Zudem kann der/die Lehrende in einem unbenoteten Setting weitaus authentischer die Rolle eines Lernbegleiters beziehungsweise einer Lernbegleiterin einnehmen, da hier für alle Beteiligten größere Rollenklarheit herrscht. Eine qualitative Befragung der involvierten Lehrenden legt nahe, dass die spezifische Lehr- und Prüfungsform der Schlüsselkompetenz-Module - in Form von E-Portfolio und Kolloquium - eine andere Qualität des Austauschs zwischen Lehrenden und Studierenden ermöglicht und die Reflexion der Lehrenden über die eigene Rolle und das Lehrhandeln anregt (Buhl/Seidl/Zeiner 2019). Es ist zu erwarten, dass sich dieser Reflexionsprozess auch positiv auf das Lehrhandeln in anderen Veranstaltungen auswirkt.

\section{Projektphase}

In den Semestern 6 und 7 wurden bei allen Bachelorstudiengängen der Fakultät Information und Kommunikation die traditionelle Form des Studiums durch eine projektbasierte Studienform ersetzt, die starke Anleihen am Konzept des Problembasierten Lernens aufweist. Anstelle von inputorientierten Veranstaltungen mit stark repetitiven Prüfungselementen sollen die Studierenden auf ihre, im Lauf des Studiums erworbenen Kompetenzen aufbauen und an der Lösung konkreter Problemstellungen die fachlichen und überfachlichen Kompetenzen erweitern. Während traditionelle Projektveranstaltungen stark fachspezifisch geprägt sind, werden die Studierenden im neuen Studienkonzept der Fakultät auch mit interdisziplinären und transdisziplinären Projekt-Problemstellungen konfrontiert, das heißt mit Problemstellungen, die unterschiedliche fachliche Perspektiven zur Lösung benötigen. Im Rahmen eines transdisziplinären Projekts behandeln die Studierenden konkrete Fragestellungen aus der Praxis in 
einem Team, in dem neben den Lehrenden und den Studierenden auch Vertreterinnen und Vertreter der Unternehmenspraxis integriert sind. Diese Projektform ist inspiriert von der aktuellen Wissenschaftsströmung der „transformativen Wissenschaft“ und ihres wesentlichen Instruments, dem Reallabor (Verband für nachhaltige Wissenschaft 2013). Die an einem transdisziplinären Projekt beteiligten Studierenden agieren damit als Wissenschaftsteams, die sich auf die spezifischen, im Verlauf eines Projekts wandelbaren Bedürfnisse des oder der Projektpartnerinnen und Projektpartner sowie der von den Transformationen betroffenen Personen einstellen müssen. Zur Bearbeitung beziehungsweise zur Lösung der Projektaufgabe müssen sie damit nicht nur über jeweils eigene Fachkompetenz verfügen, sie müssen die unterschiedlichen Fachkompetenzen auch zielführend integrieren können sowie den gesamten Lösungsprozess moderieren und begleiten.

Der Projektbereich umfasst 35 ECTS, die von den Studierenden durch eine beliebige Kombination von Projekten aus drei unterschiedlichen Projektkategorien erbracht werden können (siehe Tabelle 4). Einzige Randbedingung für die Wahl von Projekten ist, dass die Studierenden im Laufe ihres Studiums mindestens ein inter- oder transdisziplinäres Projekt erfolgreich absolviert haben müssen.

Tab. 4: Ausprägungsformen von Projektveranstaltungen.

\begin{tabular}{|c|c|c|c|c|}
\hline Projektkategorie & Dauer & SWS / ECTS & Lehrende & Fachliche Ausrichtung \\
\hline $\begin{array}{l}\text { Fachspezifische } \\
\text { Projekte }\end{array}$ & $1 \mathrm{Sem}$. & $\begin{array}{l}5 \text { ECTS / } \\
3 \text { SWS }\end{array}$ & $\begin{array}{l}\text { in der Regel ein/e } \\
\text { Lehrende/r }\end{array}$ & $\begin{array}{l}\text { Fachbezogen (Man- } \\
\text { agement, IT, Medien- } \\
\text { produktion, Medien } \\
\text { und Kultur) }\end{array}$ \\
\hline $\begin{array}{l}\text { Interdisziplinäre } \\
\text { Projekte }\end{array}$ & $1 \mathrm{Sem}$. & $\begin{array}{l}10 \text { ECTS / } \\
5 \text { SWS }\end{array}$ & $\begin{array}{l}\text { mindestenz zwei } \\
\text { Lehrende aus unter- } \\
\text { schiedlichen Fach- } \\
\text { disziplinen und/ } \\
\text { oder Studiengän- } \\
\text { gen }\end{array}$ & $\begin{array}{l}\text { Interdisziplinär (belie- } \\
\text { bige Kombination der } \\
\text { o. ä. Fachrichtungen) }\end{array}$ \\
\hline $\begin{array}{l}\text { Transdisziplinäre } \\
\text { Projekte }\end{array}$ & 2 Sem. & $\begin{array}{l}20 \text { ECTS / } \\
10 \text { SWS }\end{array}$ & $\begin{array}{l}\text { mindestens zwei } \\
\text { Lehrende aus unter- } \\
\text { schiedlichen Fach- } \\
\text { disziplinen und/ } \\
\text { oder Studiengängen } \\
\text { sowie mindestens } \\
\text { ein Praxispartner }\end{array}$ & $\begin{array}{l}\text { Transdisziplinär; Real- } \\
\text { labor (komplexe reale } \\
\text { Problemstellung; } \\
\text { Kombination unter- } \\
\text { schiedlicher Fachrich- } \\
\text { tungen) }\end{array}$ \\
\hline
\end{tabular}


Da der Projektbereich erst mit Beginn des Sommersemester 2019 gestartet ist, können noch keine verlässlichen Aussagen zum Erfolg gemacht oder fundierte Evaluationsergebnisse präsentiert werden. Eine erste Auswertung der studentischen Lehrevaluation zeigt jedoch ein hohes Maß an Zufriedenheit der Studierenden in Hinblick auf die Quantität sowie im Hinblick auf die Qualität der angebotenen Projekte. Die Studierenden betonen dabei immer wieder, den hohen Kompetenzgewinn oder den Mehrwert durch die ausgeprägte Interdisziplinarität der Veranstaltungsform Projekte.

\section{Fazit}

Mit der Vorbereitung durch die Schlüsselkompetenzmodule, dem Aufbau der Grundlagen für kreatives und wissenschaftlich fundiertes Problemlösen sowie der Entwicklung des disziplinären fachlichen Wissens werden die Voraussetzungen geschaffen, dass sich Studierende im Rahmen der interdisziplinären und transdisziplinären Projekte im sechsten und siebten Semester Herausforderungen aus Wirtschaft und Gesellschaft stellen können. So wird eine Verbindung von Third Mission der Hochschulen und der transformativen Wissenschaft hergestellt. Die ersten Projekte zu Fragen wie Wege menschengerechter Digitalisierung, Konzeption von Arbeits- und Lernräumen an Hochschulen, digitalisierte Bildung und Ausbildung oder nutzbringender Einsatz und Konzeption immersiver Technologien wie Virtual Reality und Augmented Reality, zeigen ein positives Echo bei Studierenden und Lehrenden. In den nächsten Semestern gilt es die wirtschaftlichen und gesellschaftlichen Wirkungen genauer zu evaluieren und das transformative Problemlösungspotenzial im Austausch von Hochschule und Gesellschaft weiter zu stärken.

Die erfolgreiche (Weiter-)Entwicklung von Curricula in einer sich schnell verändernden Welt hängt von mehreren Faktoren ab:

- Die Curricula müssen inhaltlich und didaktisch an aktuelle Erkenntnisse über Entwicklungen in Wirtschaft und Gesellschaft sowie der Lehr-Lernforschung anschlussfähig sein.

- Sie müssen im Hinblick auf Studien- und Prüfungsordnungen rechtssicher jedoch mit wenig administrativem Aufwand - iterativ weiterentwickelbar sein.

- Die Lehrenden müssen bereit sein, sich auf Veränderungen einzulassen, neuen Herangehensweisen auszuprobieren und sich in neue Inhalte einzuarbeiten. 
Mit dem neuen Studienmodell hat sich die Fakultät Informationen und Kommunikation der Hochschule der Medien Stuttgart auf den Weg gemacht, Lehre an die Anforderungen der Zukunft anzupassen. Neben den positiven Rückmeldungen aus Scholarship of Teaching and Learning-Projekten, Studierendenevaluationen und Industriebeiräten lässt sich eine weitere erfreuliche Wirkung beobachten: Die Lehrenden der Fakultät nehmen Lehrentwicklung immer mehr als Gemeinschaftsaufgabe wahr, diskutieren über Lehre und beteiligen sich an Experimenten. Diese Veränderung auf kultureller Ebene lässt hoffen, dass der eingeschlagene Weg - im Sinne einer kontinuierlichen iterativen Weiterentwicklung - auch in Zukunft erfolgreich weitergegangen werden kann.

\section{Literatur}

Arbeitsgruppe Curriculum 4.0 (2018): Curriculumentwicklung und Kompetenzen für das digitale Zeitalter. Thesen und Empfehlungen der AG Curriculum 4.0 des Hochschulforum Digitalisierung. Arbeitspapier Nr. 39. Berlin: Hochschulforum Digitalisierung. DOI: 10.5281/zenodo.2602541.

Buhl V.; Seidl, T.; Zeiner, K. (2019): Einfluss eines ePortfolio-Einsatzes in der Lehre auf Selbstverständnis und Perspektiven der Lehrenden. Die Hochschullehre 5, 249-264.

Hochschulrahmengesetz (HRG). http://www.landesrecht-bw.de/jportal/?quelle=jlink\& docid=BJNR001850976\&psml=bsbawueprod.psml\&max=true.

Mildenberger, U.; Vonhof, C. (2020): Neues Studienmodell und organisatorische Herausforderungen. Wege zu einer transformativen Fakultät. In: R. Stang; A. Becker (Hrsg.): Zukunft Lennwelt Hochschule. Perspektiven und Optionen für eine Neuausrichtung. Berlin; Boston: De Gruyter Saur, 26-34.

Schaper, N.; Hilkenmeier, F. (2013): Umsetzungshilfen für kompetenzorientiertes Prüfen. https://www.hrk-nexus.de/fileadmin/redaktion/hrk-nexus/07-Downloads/07-03-Material/zusatzgutachten.pdf.

Schütz-Pitan, J.; Seidl, T.; Hense, J. (2019): Wirksamkeit eines flächendeckenden ePortfolioEinsatzes in der Hochschullehre. Einflussfaktoren auf den Kompetenzerwerb. Die Hochschullehre 5, 769-796.

Seidl, T.; Vonhof, C. (2017): Agile Prinzipien. Was kann die Studiengangsentwicklung davon lernen? Synergie. Fachmagazin zur Digitalisierung in der Lehre 3, 22-25.

Van den Berk, I.; Petersen, K.; Schultes K.; Stolz, K. (Hrsg.) (2016): Studierfähigkeit. Theoretische Erkenntnisse, empirische Befunde und praktische Perspektiven. Hamburg: Universität Hamburg., https://www.universitaetskolleg.uni-hamburg.de/publikationen/uk-schriften-015.pdf.

Verband für nachhaltige Wissenschaft (2013): Was ist eine „transformative Wissenschaft“? http://nachhaltigewissenschaft.de/2013/04/08/transformative-wissenschaft-15731128/.

Wildt J.; Wildt, B (2011): Lernprozessorientiertes Prüfen im „Constructive Alignment“. In: B. Berendt; H.-P. Voss; J. Wildt (Hrsg.): Neues Handbuch Hochschullehre. Teil H: Prüfungen und Leistungskontrollen. Weiterentwicklung des Prüfungssystems in der Konsequenz des Bologna-Prozesses. Berlin: DUZ, 1-46. 